\title{
Validación empírica del modelo conceptual de Gestión Empresarial para las MYPES de la Región Lambayeque, 2018
}

\section{Empirical validation of the conceptual model of Business Management for the MYPES of the Lambayeque Region, 2018}

Jorge A. Mundaca $\mathrm{G}^{1}$ y Jorge A. Huarachi $\mathrm{C}^{2}$

\begin{abstract}
RESUMEN
El estudio parte del modelo conceptual de gestión empresarial. Se utilizó el método deductivo para determinar la correlación y la influencia de las variables categóricas del modelo conceptual. Se determinaron las brechas por cada variable del modelo. El problema de investigación: ¿Se valida empíricamente el Modelo Conceptual de Gestión Empresarial para las MYPES de la Región Lambayeque? ¿Cuál es el nivel de validación que se puede lograr? ¿Qué aporta al conocimiento y qué aplicabilidad se le debe dar al modelo validado? El objetivo general fue determinar la validación empírica del Modelo Conceptual. Se confirman las hipótesis planteadas en el estudio, demostrado por una alta correlación y nivel de significancia, prueba Kaiser - Meyer - Olkin (KMO) elevado. No se confirman las hipótesis alternativas de las variables moderadoras. Los resultados validaron el Modelo Conceptual según lo planteado en los objetivos específicos, adicionalmente, el aporte teórico precisa el desarrollo de líneas de investigación, estrategias en los procesos de formación y desarrollo empresarial que debe asumir PRODUCE y el Centro de Desarrollo Empresarial - CDE, USAT como órgano operativo.
\end{abstract}

Palabras clave: Valores del empresario, capital social organizacional, cultura organizacional, orientación emprendedora, orientación al mercado.

\begin{abstract}
The study starts from the conceptual model of business management. The deductive method was used to determine the correlation and influence of the categorical variables of the conceptual model. Gaps were determined for each model variable. The research problem: Is the Conceptual Model of Business Management for MYPES of the Lambayeque Region empirically validated? What is the level of validation that can be achieved? What contributes to knowledge and what applicability should be given to the validated model? The general objective was to determine the empirical validation of the Conceptual Model. The hypotheses raised in the study are confirmed, demonstrated by a high correlation and level of significance, Kaiser test - Meyer - Olkin (KMO) elevated. The alternative hypotheses of the moderating variables are not confirmed. The results validated the Conceptual

Model as set out in the specific objectives; in addition, the theoretical contribution requires the development of lines of research, strategies in the training and business development processes that must be assumed by PRODUCE and the Center for Business Development - CDE, USAT as an operating body.
\end{abstract}

Keywords: Values of the entrepreneur, organizational social capital, organizational culture, entrepreneurial orientation, market orientation.

\footnotetext{
${ }^{1}$ Universidad Católica Santo Toribio de Mogrovejo, Chiclayo, Perú. Email: jmundaca@usat.edu.pe

${ }^{2}$ Universidad Católica Santo Toribio de Mogrovejo, Chiclayo, Perú. Email: j.huarachi@usat.edu.pe
} 


\section{INTRODUCCIÓN}

Las microempresas en el Perú representan el $96.1 \%$ de las empresas en el Perú, las pequeñas empresas el $3.3 \%$ y las medianas y grandes empresas el $0.6 \%$. Es muy pequeño el tamaño de las micro y pequeñas empresas y reflejan un marcado aislamiento respecto de las medianas y grandes empresas el cual se incrementa año a año (Herrera, 2011).

La presente investigación permite ampliar las líneas de investigación vigentes a campos poco explorados que tienen que ver con el desarrollo local a través del desarrollo empresarial de las Micro y pequeñas empresas que son la gran mayoría en nuestro país. Temas de investigación que tienen que ver con el aislamiento del sector frente a las medianas y grandes empresas que tienen un desarrollo natural en ascenso y oportunidad empresarial a diferencia de las MYPES que colapsan en un70\% antes del tercer año de creadas. Otro tema importante relacionado a lo anterior tiene que ver sobre cómo desarrollar emprendimientos dinámicos rápidamente escalables considerando que un $40 \%$ de los interesados en desarrollar emprendimientos son profesionales egresados de las universidades u otros centros superiores. De esta manera facilitar el camino a emprendimientos que no sean por necesidad sino por un desarrollo empresarial basado en la innovación y el capital humano.

Objetivo general: Determinar la validación empírica del Modelo Conceptual de Gestión Empresarial para las MYPES de la Región Lambayeque (Mundaca, 2018).

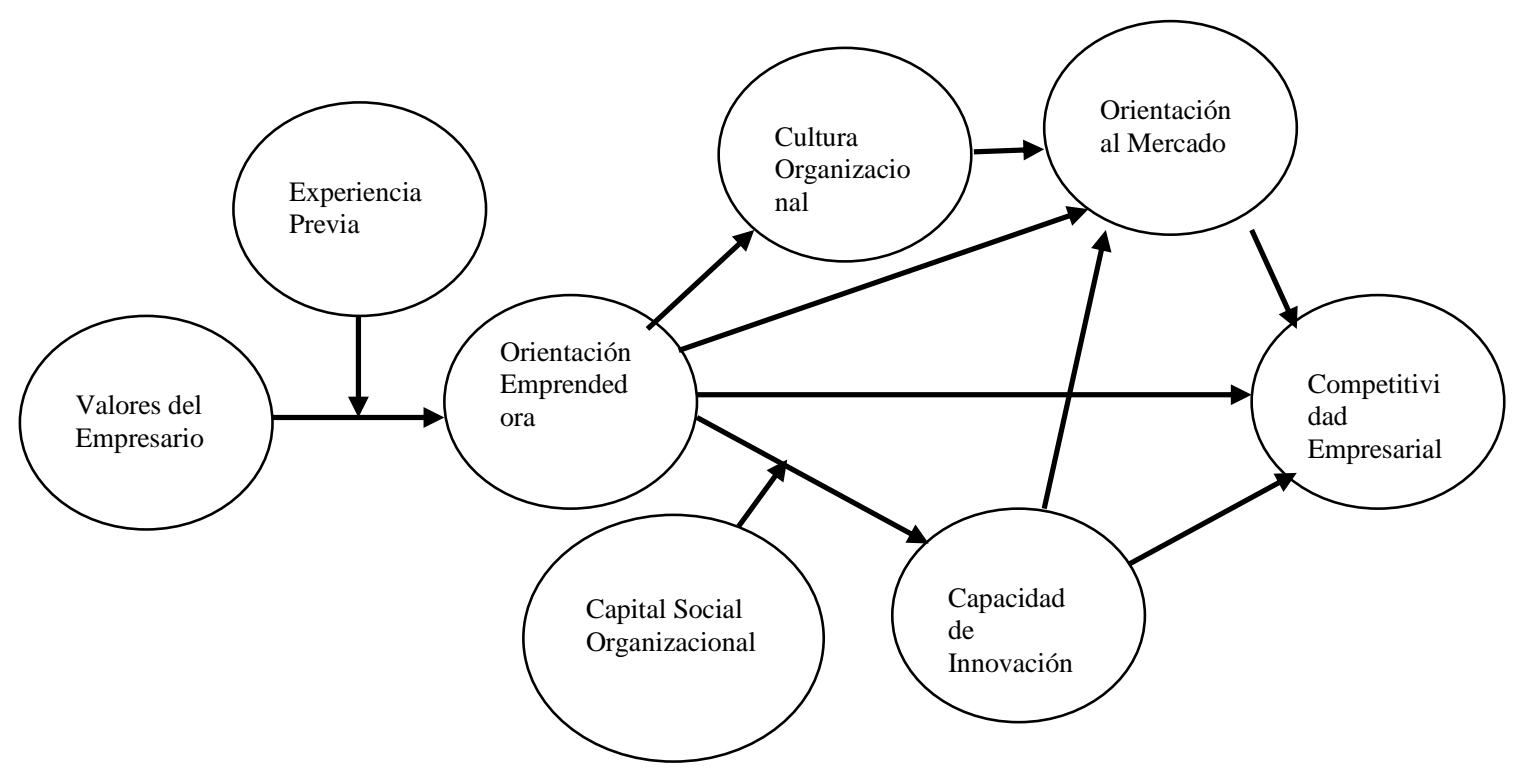

Figura. 1 Modelo Conceptual de Gestión Empresarial

Fuente: Mundaca (2018)

El modelo conceptual basa su desarrollo en la clasificación de Global Entrepreneurship Monitor GEM: Los empresarios potenciales, iniciados, establecidos y consolidados, los cuales están influenciados por el entorno familiar por un lado y/o por las oportunidades reales de mercado que se le 
presentan. Los valores familiares inciden en los valores del empresario, los mismos que pueden incidir negativamente o positivamente en el nacimiento de un emprendimiento sea por subsistencia o por oportunidad: tradicional o dinámico. La orientación emprendedora es inducida por los valores empresariales, creencias empresariales y prácticas empresariales, que son los componentes de la cultura organizacional que oriente a la futura empresa. El capital social organizacional considera la confianza, el compromiso y el beneficio mutuo, expresándose en los factores internos y externos de la empresa frente al entorno. El capital social se consideró como una variable moderadora entre la orientación emprendedora y la capacidad de innovación. La experiencia previa actúa como moderadora entre los valores del empresario y la orientación emprendedora. La cultura organizacional debe tener cuatro orientaciones: orientación al cliente, orientación a la competencia, orientación a la coordinación interfuncional y orientación a la rentabilidad. La cultura organizacional en esencia debe priorizar la orientación al cliente por ser quien da sostenibilidad al negocio. Otras orientaciones son estrategias para garantizar una información real que, de lectura del comportamiento del mercado para garantizar capacidad de respuesta, la repetición de la compra, la organización interfuncional coordinada y el rendimiento del negocio. La competitividad empresarial es la variable vinculada al rendimiento empresarial y, según el modelo, se sustenta en la orientación al mercado y la capacidad de innovación. A su vez, la orientación al mercado requiere de la innovación ya que la propuesta de valor es en esencia innovación e influye en el comportamiento emocional de compra de los clientes. La competitividad empresarial es el resultado del logro de indicadores de desempeño innovador, por tanto, deben priorizarse las intervenciones para mejorar esos indicadores de desempeño en cualquiera de los tipos de emprendimiento. Los indicadores de desempeño y rendimiento tienen que ver con la innovación de producto, de procesos, mejoras organizacionales y de mercadotecnia que permiten generar ventajas competitivas, adicionando el desarrollo de estrategias competitivas tecnológicas para lograr la mayor ventaja diferencial frente a la competencia.

Para (Knoppen, 2015) los valores representan las respuestas que las personas y los grupos deben dar a tres requisitos universales: (a) las necesidades de los individuos en su condición de seres biológicos, (b) la coordinación de las acciones sociales y (c) el funcionamiento correcto y la supervivencia de los grupos. Se resumen en 10 valores o tipos motivacionales. De los valores del fundador se derivan los valores empresariales, las creencias empresariales y las prácticas empresariales que forman la cultura organizacional. Se pueden clasificar en dos grandes clases de valores, que muestran la estructura propia. (Steckerl, 2005) 
La cultura organizacional es la fuente de ventaja competitiva; recurso valorable raro e imperfectamente inimitable. Raro desde el aspecto de sus atributos y características que deberán ser poco comunes para que una empresa sea identificada y posicionada en el mercado de manera específica (Páramo, 2001). Según Shumpeter (1934) la innovación se entiende en un sentido amplio e identificó el comportamiento emprendedor con una gran variedad de innovaciones empresariales que pueden tomar la forma de nuevos productos, procesos, mercados, materias primas, e incluso diferentes formas organizativas. Según Narver y Slater,(1994), una organización orientada al mercado es aquella que desarrolla comportamientos coordinados de las diferentes funciones de la organización dirigidos a buscar y recoger información de los consumidores, de la competencia y del entorno; disemina dicha información por la organización y diseña e implementa una respuesta acorde con la información obtenida, basándose para ello en la identificación y construcción de capacidades distintivas de la organización, con el objetivo de satisfacer a los consumidores proporcionándoles un valor superior. (Blesa y Ripollés, 2005). La capacidad de innovación, habilidad de la empresa para generar y transformar ideas y conocimiento con el propósito de aprovechar las oportunidades de mercado. Implica la utilización de un nuevo conocimiento o un nuevo uso o una combinación de conocimientos existentes. El nuevo conocimiento puede haber sido generado por la empresa innovadora en el curso de sus actividades de innovación (es decir con la I $+\mathrm{D}$ interna) o adquirido externamente a través de determinados canales (es decir comprando la nueva tecnología). La utilización de un nuevo conocimiento o de una combinación de conocimientos existente requiere esfuerzos innovadores por la empresa que pueden ser diferenciados de las rutinas habituales. La empresa innovadora puede definir nuevos conceptos de producto, procesos, métodos de comercialización o cambios organizativos. (Manual de Oslo $3^{\circ}$ Edición, 2006). El Capital Social Organizacional - CSO, es un recurso que refleja la calidad de las relaciones internas de una empresa. El CSO interno se produce por las relaciones sociales internas; ellas comprenden las vinculaciones entre el equipo directivo de una empresa y sus empleados y la existente entre los empleados entre sí. El CSO externo se construye a partir de la relación entre los miembros de la empresa y sus partes interesadas externas (Román, Gómez y Smida, 2013). Se evidencia una falta de consenso en la medición del rendimiento emprendedor o de la orientación al mercado. Las variables más comúnmente utilizadas en la literatura de emprendimiento son aquellas cuya información es más fácil de recoger. En consecuencia, la medida de rendimiento más utilizada es el crecimiento, especialmente el crecimiento de las ventas. Una perspectiva alternativa consiste en reconocer la múltiple dimensionalidad del rendimiento.

Los modelos de desarrollo existentes se formulan alrededor de la competitividad empresarial como variable dependiente. Tal es el caso de Martínez, Charterina y Araujo (2010) que desarrolla el modelo 
explicativo de la competitividad empresarial a partir de factores internos a la empresa. Para ello se identifican los factores de competitividad más importantes en el caso de las empresas manufactureras vascas, así como las variables que permiten caracterizarlos, utilizando técnicas cualitativas basadas en entrevistas con directivos. Los factores resultantes son las capacidades directivas, las capacidades de innovación, las capacidades de marketing y las capacidades de calidad. Este modelo otorga una importancia especial a las capacidades directivas al plantear este factor como precedente del resto de capacidades de la empresa.

Como limitación, en vista que aún no se han realizado investigaciones empíricas que permitan medir los valores de la familia, se han tomado los Valores Humanos de Schwartz ampliamente utilizados en investigaciones sobre iniciativas de emprendimiento.

\section{MATERIALES Y MÉTODOS}

\section{Población y muestra}

La Población en estudio son los Micro y Pequeños Empresarios de la Región Lambayeque, seleccionados aleatoriamente y por sectores empresariales, de los cuales se tomó a toda la población de empresas establecidas y consolidadas con más de un año de vigencia en el mercado como criterio de inclusión. La metodología desarrollada parte del interés por incluir a las MYPEs de los tres sectores representativos: Manufactura, comercio y servicios que son a los que atiende el Centro de Desarrollo Empresarial - PRODUCE en la Universidad Católica Santo Toribio de Mogrovejo - USAT. No se incluyen a potenciales emprendedores a pesar que registran un $60 \%$ de visitas al centro por no ser el objeto de estudio. La muestra calculada fue de 382 empresas de los sectores manufactura, comercio y servicios. Instrumento cuestionario y técnica encuestas. El muestreo fue no estratificado.

El objeto de estudio es el Modelo Conceptual de Gestión Empresarial. El nivel de investigación es explicativo, el método es deductivo y el tipo de investigación es cuantitativa analítica. En cuanto al diseño de investigación: no experimental, analítica explicativa, transversal, orientada a generalizar y obtener conclusiones y no existe manipulación de variables. Preferente utilización del método hipotético deductivo, estudio sobre muestras grandes de sujetos seleccionados por método de muestreo probabilístico, aplicación de test y medidas objetivas del comportamiento, la consideración del investigador como elemento externo al objeto que se investiga, aplicación de técnicas estadísticas en el análisis de datos, generalización de los resultados, etc.

Se aplicaron siete instrumentos validados en la literatura: Valores del Empresario (Knoppen y Saris, 2015, Cultura Organizacional (Vallejos - Martos, 2011), Orientación Emprendedora (Blesa y Ripollés, 
2005), Orientación al Mercado (Blesa y Ripollés, 2005), Capacidad de Innovación (Manual de Oslo, 2006) e Instituto Nacional de Estadísticas de Chile (2015), Capital Social Organizacional (Roman, Gómez y Smida, 2013) y Competitividad Empresarial (Blesa y Ripollés, 2005)

\section{Técnicas de procesamiento y análisis de datos}

La matriz de datos obtenida representó el punto de partida para el análisis, utilizando un programa de análisis estadístico SPSS 25 y el Análisis Factorial Confirmatorio en vista que se buscan patrones entre variables y contarse con teorías respecto de tales patrones que se desean probar (Vogt, Vogt, Gardner y Haeffele, 2014., pág. 333). La data base para el Análisis Factorial es la Matriz de Correlación o Matriz de covarianza o ambos con el fin de que la información sea útil para el Análisis Factorial (Pág. 334). Para que el Análisis Factorial sea efectivo la correlación entre variables debe ser alta y el tamaño de la muestra debe ser representativa de la población (Pág. 335), por ello, se determinaron las matrices de correlación, matrices de varianzas y componentes, prueba KMO y la prueba de esfericidad de Barlett; análisis de regresión lineal múltiple. Solo después que se ha aplicado el Análisis Factorial se podrá conocer las comunalidades y el número de variables por factor, es decir, la varianza es explicada por los factores comunes de una variable y se puede definir el tamaño exacto de la muestra (Pág. 336). Normalmente un tamaño de muestra de 500 sería suficiente en la mayoría de los casos, pero un tamaño no menor de 300 es aceptable (Pág. 336). Para el análisis confirmatorio también se procedió previamente al análisis multivariable de normalidad, de correlación (Spearman) y análisis de colinealidad / multicolinealidad, luego se procedió al análisis de componentes. Finalmente, al no ser significativo el análisis de regresión múltiple se procedió al análisis de regresión simple de acuerdo a lo inferido en el Modelo Conceptual de Gestión Empresarial.

La validez del contenido y de constructo se realizó en muestra piloto que fue validada por expertos con Línea de Investigación en Emprendimiento y Competitividad Empresarial en un número de tres. El número de expertos consultados debe oscilar entre 3 y 10 (Vara, 2015, pág. 306). Para la fiabilidad de los instrumentos se utilizó el método de Alfa de Cronbach dirigida a muestra piloto de 18 empresas y a la totalidad de la muestra.

\section{RESULTADOS}

El análisis de confiabilidad arrojo una fuerte consistencia interna. Se permitieron las condiciones más adecuadas para su aplicación: capacitación a los encuestadores quienes contaron con credenciales del Centro de Desarrollo Empresarial de la USAT, siendo el monitoreo riguroso y evidenciado. Se utilizaron Instrumentos ya validados y, como criterio de inclusión, solo se encuestaron empresas con más de un año de antigüedad para garantizar la uniformidad de las respuestas. El valor obtenido es de 
0.986. Se considera que existe una buena consistencia interna cuando el valor de Alfa es superior a 0.7 (Vara, 2015, pág. 305).

Para el análisis de normalidad (Kolmogorov - Smirnoff), el nivel de significancia está por debajo de 0.5 por tanto, todas las variables de estudio siguen una distribución normal. Asimismo, el supuesto de linealidad se cumple. En ninguno de los casos existen problemas de multicolinealidad ni colinealidad por los valores de las correlaciones entre variables. Se utilizó el coeficiente Spearman y no Pearson por ser un estudio multivariable y éstas se encuentran en escala ordinal (de 0 a 4 ).

Tabla 1. Prueba de KMO y Barlett

\begin{tabular}{lll}
\hline \multicolumn{2}{l}{ Medida Kaiser-Meyer-Olkin de acuación de muestreo } & 0.970 \\
\hline & Aprox Chi-cuadrado & 64214.444 \\
Prueba de esfericidad de Bartlett & gl & 4560 \\
& Sig. & 0.000 \\
\hline
\end{tabular}

El Índice KMO mide la adecuación de la muestra. Indica qué tan apropiado es aplicar el Análisis Factorial. Los valores entre 0.5 y 1 indican que es apropiado aplicarlo. En este caso, el valor KMO es 0.970 bastante alto. Por tanto, se continúa con el análisis y no se justifica realizar el análisis de componentes rotados. El Test de Esfericidad de Bartlett: Se utiliza para probar la Hipótesis Nula que afirma que las variables no están correlacionadas en la población. Es decir, comprueba si la matriz de correlaciones es una matriz de identidad. Se puede dar como válidos aquellos resultados que nos presenten un valor elevado del test y cuya fiabilidad sea menor a 0.05 . El nivel de significancia es menor a 0.05. En este caso se rechaza la Hipótesis Nula y se continúa con el Análisis Factorial. La matriz de correlación demuestra que el determinante de la matriz es bajo por lo que se demuestra que la intercorrelación entre variables es alta. La relación entre las variables Valores del Empresario y Competitividad Empresarial no es significativa. A nivel del modelo en general si es significativa. Por tanto, se puede continuar con el análisis factorial. Se determinaron 11 componentes que explica, la mayor cantidad de la varianza en la matriz de correlación. Los componentes del estudio fueron 7. Respecto del análisis de regresión lineal múltiple del modelo, $\mathrm{R}^{2}$ ajustado es 0.406 (40.6\%) que significa una correlación moderada del modelo, desde la variable dependiente hacia las variables independientes. El nivel de significancia es alto. En el análisis de coeficientes, los niveles de significancia, asumiendo regresión lineal múltiple están por encima de 0.05 , por tanto, a pesar que la correlación es alta en el modelo no se puede concluir que exista una relación de causalidad entre las variables independientes y dependiente a excepción de la variable Capacidad de innovación que si 
determina causalidad sobre la variable competitividad empresarial. Las variables: (A) Valores del empresario, (B) Capital Social Organizacional, (C) Cultura Organizacional de la Empresa, (D) Orientación al Mercado de la empresa, (E) Orientación Emprendedora de la Empresa, (F) Capacidad de Innovación de la Empresa, (G) Competitividad Empresarial. Los resultados de la regresión simple:

Tabla 2. Análisis de la Regresión Lineal Simple entre Variables

\begin{tabular}{|c|c|c|c|c|}
\hline Orden & $\begin{array}{c}\text { Variables } \\
\text { Independiente - Dependiente }\end{array}$ & Sig. & $R^{2}$ ajustado & $\begin{array}{l}\text { Relación de } \\
\text { Causalidad }\end{array}$ \\
\hline 1 & $\begin{array}{l}\text { Capital Social Organizacional - Cultura Organizacional de la } \\
\text { Empresa }\end{array}$ & 0.000 & 0.861 & $Y=0.345+0.928 X$ \\
\hline 2 & $\begin{array}{l}\text { Cultura Organizacional - Orientación al Mercado de la } \\
\text { Empresa }\end{array}$ & 0.000 & 0.774 & $Y=0.152+0.880 X$ \\
\hline 3 & $\begin{array}{l}\text { Capital Social Organizacional - Orientación al Mercado de la } \\
\text { Empresa }\end{array}$ & 0.000 & 0.767 & $Y=0.285+0.876 X$ \\
\hline 4 & $\begin{array}{l}\text { Orientación Emprendedora de la Empresa -Orientación al } \\
\text { Mercado }\end{array}$ & 0.000 & 0.519 & $Y=0.768+0.721 X$ \\
\hline 5 & $\begin{array}{l}\text { Orientación Emprendedora de la Empresa -Capital Social } \\
\text { Organizacional de la Empresa }\end{array}$ & 0.000 & 0.475 & $Y=0.932+0.690 X$ \\
\hline 6 & $\begin{array}{l}\text { Orientación Emprendedora de la Empresa - Cultura } \\
\text { Organizacional de la Empresa }\end{array}$ & 0.000 & 0.447 & $Y=1.25+0.670 X$ \\
\hline 7 & $\begin{array}{l}\text { Capacidad de Innovación de la Empresa - Competitividad de } \\
\text { la Empresa }\end{array}$ & 0.000 & 0.407 & $Y=0.294+0.639 X$ \\
\hline 8 & $\begin{array}{l}\text { Orientación Emprendedora de la Empresa - Capacidad de } \\
\text { Innovación de la Empresa }\end{array}$ & 0.000 & 0.251 & $Y=1.333+0.502 X$ \\
\hline 9 & $\begin{array}{l}\text { Capacidad de Innovación de la Empresa- Orientación al } \\
\text { Mercado de la Empresa }\end{array}$ & 0.000 & 0.181 & $Y=1.393+0.428 X$ \\
\hline 10 & $\begin{array}{l}\text { Capital Social Organizacional de la Empresa - Capacidad de } \\
\text { Innovación de la Empresa }\end{array}$ & 0.000 & 0.147 & $Y=1.552+0.386 X$ \\
\hline 11 & $\begin{array}{l}\text { Cultura Organizacional de la Empresa -Capacidad de } \\
\text { Innovación de la Empresa }\end{array}$ & 0.000 & 0.146 & $Y=1.516+0.384 X$ \\
\hline 12 & $\begin{array}{l}\text { Orientación Emprendedora de la Empresa -Competitividad } \\
\text { Empresarial }\end{array}$ & 0.000 & 0.071 & $Y=1.538+0.271 X$ \\
\hline 13 & $\begin{array}{l}\text { Valores del Empresario - Orientación Emprendedora de la } \\
\text { Empresa }\end{array}$ & 0.000 & 0.058 & $Y=-0.686+0.245 X$ \\
\hline 14 & $\begin{array}{l}\text { Orientación al Mercado de la Empresa -Competitividad } \\
\text { Empresarial }\end{array}$ & 0.000 & 0.054 & $Y=1.626+0.238 X$ \\
\hline 15 & Valores del empresario - Competitividad empresarial & 0.523 & No significativo & \\
\hline
\end{tabular}

No está considerado A - G por cuanto la correlación no es significativa, pero se mantiene en el modelo por la correlación significativa $\mathrm{A}-\mathrm{E}$. 


\section{DISCUSIÓN}

El estudio permitió determinar que las variables Experiencia Previa y Capital Social Organizacional no cumplen un rol moderador. El Capital Social Organizacional cumple un rol mediador entre las variables Orientación Emprendedora y Capacidad de Innovación, Cultura Organizacional y Orientación al Mercado de la Empresa.

La experiencia previa no se ha considerado en el modelo, pero analizando su relación con los años de experiencia en el negocio la correlación resulta significativa. En vista que los años de experiencia no se han considerado en el modelo queda para estudios posteriores incluir esta relación que es importante a la hora de establecer políticas de intervención para el desarrollo de los sectores considerando esta relación.

Todas las variables del Modelo Conceptual a excepción de Experiencia previa se mantienen y se ha podido validar el modelo a excepción de las variables moderadoras y determinar la real participación que cumple cada una de ellas en el Modelo Empírico (Ver Figura 2).

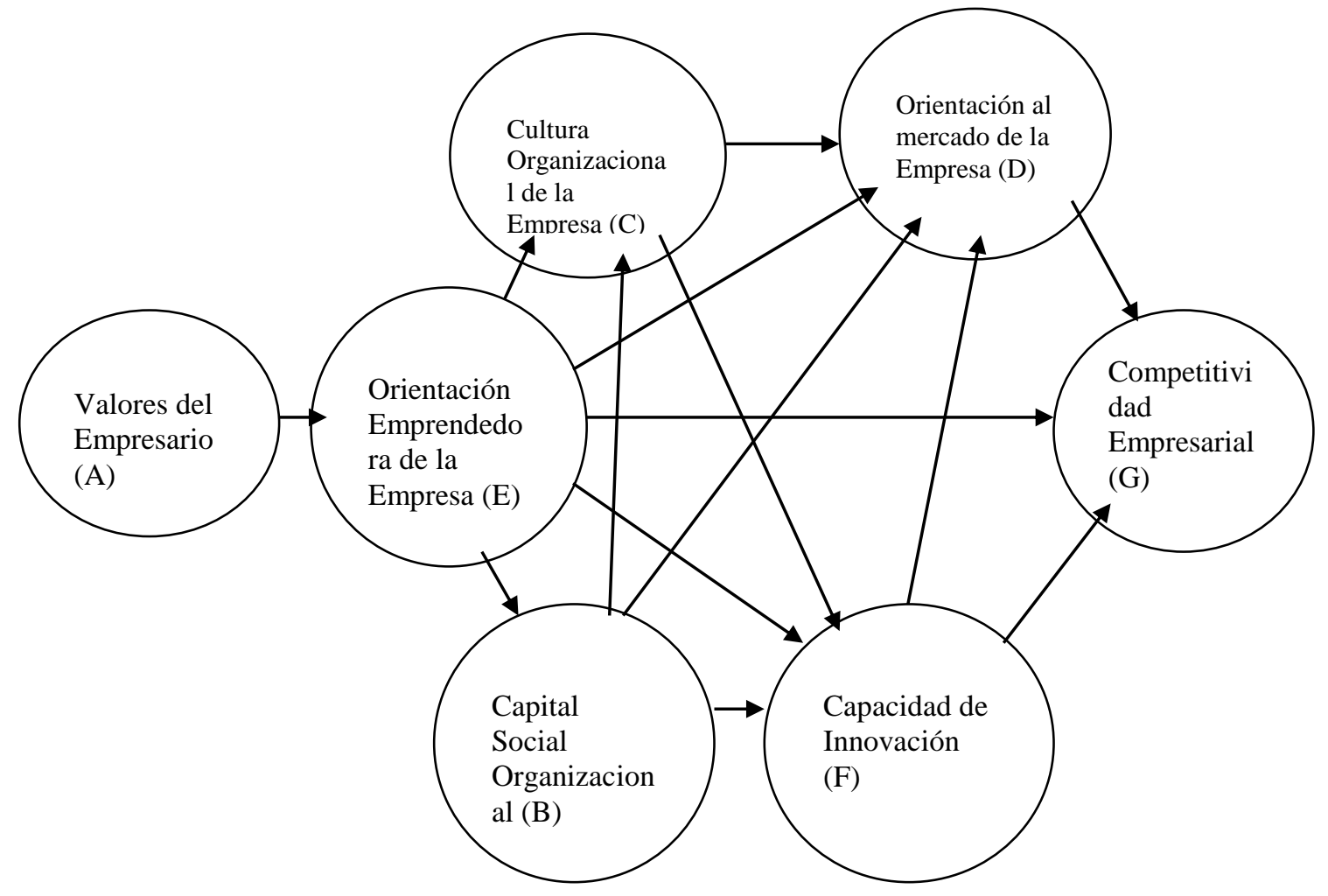

Figura 2. Modelo validado de Gestión Empresarial MYPES Región Lambayeque

El Modelo empírico en su generalización tiene una validez hasta la correlación multivariable más no a nivel de regresión múltiple o causalidad por su baja significancia estadística en vista de tratarse de 
variables no latentes. Se determina regresión múltiple entre capacidad de innovación y competitividad empresarial lo que hace que estas variables sean centrales del modelo evaluado.

\section{CONCLUSIONES}

El análisis de regresión simple permite dar respuesta a los objetivos específicos planteados en la investigación.

El modelo permite facilitar una visión sistémica de la gestión Empresarial donde todas las variables en estudio tienen una alta correlación.

Los resultados permitieron clasificar las variables y determinar las brechas resultantes:

Tabla 3. Brechas por variables

\begin{tabular}{|c|c|c|c|c|c|}
\hline Variable & $\begin{array}{c}\text { Sin } \\
\text { Importancia }\end{array}$ & $\begin{array}{c}\text { Poca } \\
\text { Importancia }\end{array}$ & $\begin{array}{c}\text { Mediana } \\
\text { Importancia }\end{array}$ & $\begin{array}{c}\text { TOTAL } \\
\%\end{array}$ & Tipo de Brecha \\
\hline Valores del Empresario & 0.3 & - & 0.3 & 1 & No Tecnológica \\
\hline $\begin{array}{l}\text { Capital Social Organizacional Interno } \\
\text { y Externo }\end{array}$ & 20 & 2.3 & 4.5 & 28 & No Tecnológica \\
\hline $\begin{array}{l}\text { Cultura Organizacional de la } \\
\text { Empresa }\end{array}$ & 19.5 & 1.8 & 3.5 & 25 & No tecnológica \\
\hline $\begin{array}{l}\text { Orientación al Mercado de la } \\
\text { Empresa }\end{array}$ & 21.1 & 2 & 5.8 & 29 & Tecnológica \\
\hline $\begin{array}{l}\text { Orientación Emprendedora de la } \\
\text { Empresa }\end{array}$ & 20.6 & 1.5 & 10 & 32 & No Tecnológica \\
\hline $\begin{array}{l}\text { Capacidad de innovación de la } \\
\text { Empresa }\end{array}$ & 11 & 9 & 27.3 & 47 & Tecnológica \\
\hline $\begin{array}{l}\text { Competitividad Empresarial de la } \\
\text { Empresa }\end{array}$ & 26.1 & 3.5 & 13.8 & 43 & $\begin{array}{l}\text { Variable } \\
\text { Resultado }\end{array}$ \\
\hline Promedio significativo & & & & $34 \%$ & \\
\hline
\end{tabular}

Según la Tabla 3, los resultados arrojan que prevalecen las variables y brechas no tecnológicas en el modelo y estas tienen que ver principalmente con capacidades organizativas y el capital humano.

Se determina que la capacidad de innovación de producto y de procesos es una variable tecnológica con mayor brecha que refleja y confirma la realidad de las MYPES en el Perú y explican su débil sostenibilidad en el mercado.

Los resultados en general, nos llevan a interpretar la importancia del capital humano para desarrollar una cultura organizacional orientada al mercado con innovación y desarrollo tecnológico; su condición emprendedora para desarrollar una capacidad de innovación que es otro eje y vía para lograr la competitividad empresarial y la sostenibilidad. 
A partir de las brechas, los procesos de mejora del rendimiento y competitividad empresarial no pueden ir aislados de los procesos de desempeño donde el rol del capital humano es fundamental a pesar de la tecnología empleada.

Los ejes fundamentales del desarrollo empresarial son la competitividad empresarial y la innovación tecnológica.

La competitividad empresarial como rendimiento se sustenta en la innovación y el capital humano e inciden en la producción, productividad y rentabilidad empresarial.

El modelo empírico es generalizable hasta un nivel de correlación que puede utilizarse para la generación de políticas públicas y Programas de Desarrollo donde todas las variables son importantes iniciándose por los Valores del Empresario que deben condicionar la orientación emprendedora con un comportamiento ético.

La capacidad de innovación como eje de desarrollo empresarial se sustenta en la investigación y el desarrollo tecnológico que son los impulsores del emprendimiento dinámico como alternativa para reducir los emprendimientos por sobrevivencia.

La aplicación de I+D+i es la mejor herramienta de intervención por parte del estado y de las universidades para permitir mejores intervenciones para desarrollar las MYPES.

La participación del estado, las universidades, sector empresarial y sociedad civil son clave para desarrollar una Agenda de Innovación Regional que permita un desarrollo dinámico, disruptivo y escalable de las MYPEs en la Región Lambayeque.

\section{REFERENCIAS BIBLIOGRÁFICAS}

Blesa, A. y Ripollés, M. (2005). Relación entre la orientación al mercado y la Orientación emprendedora. Revista Europea de Dirección y Economía de la Empresa, 14, 3 (2005), 165 180, Madrid.

Herrera, B. (2011). Análisis estructural de las MYPEs y PYMEs. Revista de la Facultad de Ciencias Contables 18 (35) 69-89 (2011). UNMSM, Lima, Perú.

Instituto Nacional de Estadística (2015). 9na Encuesta de Innovación de Empresas. Departamento de estadísticas Económicas, Chile

Knoppen, D. y Saris, W. (2015). Evaluation of the portrait values questionnaire using SEM: A new ESS proposal. Paper prepared for the QMSS2 seminar at Bolzano (Bozen), Italy, June 11-12, 2009. 
Martínez, R.; Charterina, J. y Araujo, A. (2010). Un modelo causal de competitividad empresarial planteado desde la visión de la empresa basada en recursos: capacidades directivas, de innovación, marketing y calidad. Investigaciones Europeas de Dirección y Economía de la Empresa Vol. 16, $N^{\circ}$ 2, 2010, pp. 165-18

Manual de Oslo (2006). Guía para la recogida e interpretación de datos sobre innovación. Tercera edición, OCDE - EUROSTAT

Mundaca, J. (2018) Modelo Conceptual de Desarrollo Empresarial: Caso Centro de Desarrollo Empresarial. Tesis Doctoral en Bienestar y Desarrollo Local, Universidad Católica Santo Toribio de Mogrovejo, Chiclayo.

Páramo, D. (2001). Hacia la construcción de un modelo de cultura organizacional orientada al mercado, Revista Colombiana de Marketing, 2, 2, junio, 2001.

Román, R.; Gómez, A. y Smida, A. (2013). El capital Social organizacional de la pequeña empresa innovadora. Un ensayo de medición en las ciudades de Cali y Medellín. Estudios Gerenciales 29 (2013) 356-367. Universidad ICESI. Elsevier, España.

Slater, S. y Narver, J. (1994). Market Orientation, Customer Value, and Superior Performance. Business Horizons, 1994, 37, issue 2, 22-28, USA.

Steckerl, V. (2006). Modelo explicativo de una empresa familiar que relaciona valores del fundador, cultura organizacional y orientación al mercado. Pensamiento \& Gestión, núm. 20, julio, 2006, pp. 194-215. Universidad del Norte Barranquilla, Colombia.

Vara, A. (2015). 7 Pasos para elaborar una Tesis. Cómo elaborar una Tesis paraa Ciencias Administrativas, Finanzas, Ciencias Sociales y Humanidades. Editorial MACRO. Lima, Perú

Vallejo - Martos, M. (2011). The organizational culture of family firms as a key factor of competitiveness. Journal of Business Economics and Management, 12:3, 451-481

Vogt, W.; Vogt, E.; Gardner, D. y Haeffele, L. (2014). Selecting the rights analyses for your data: Quantitive, Quality Methods. Editorial The Guilford Press. New York, USA. 